\title{
Evaluating the Calibration of Aqua MODIS Bands 33, 35, and 36 during Blackbody Warm-up Cool-down Events
}

\author{
Yonghong $\mathrm{Li}^{\mathrm{a}}$, Aisheng $\mathrm{Wu}^{\mathrm{a}}$, Xiaoxiong Xiong \\ aScience Systems and Applications Inc., 10210 Greenbelt Road, Lanham, MD 20706, USA \\ ${ }^{b}$ Sciences and Exploration Directorate, NASA/GSFC, Greenbelt, MD 20771, USA
}

\begin{abstract}
Aqua MODIS is the second MODIS instrument of NASA's Earth Observation System and has operated for over sixteen years since its launch in 2002. MODIS has sixteen thermal emissive bands (TEBs) located on two separate cold focal plane assemblies (CFPA). The TEBs are calibrated every scan using observations of an onboard blackbody (BB) and a space view port. Low saturation temperatures (Tsat) of Aqua MODIS bands 33, 35, and 36 cause these bands to saturate when the BB temperature is higher than their Tsat values during a BB warm-up cool-down (WUCD) cycle, therefore impacting the ability to perform nominal calibration. In addition, starting from around 2006, the CFPA temperature showed gradual variation from its nominally-controlled operating temperature due to a loss of its radiative cooler margin and the magnitude of its fluctuation reaching a maximum in 2013. The MODIS Characterization Support Team currently uses a correction that is dependent on the CFPA temperature to provide a gain estimate for the saturated scans during the BB WUCD. This gain estimation has been implemented in the Aqua MODIS Collection 6 (C6) and C6.1 L1B products. This paper evaluates the quality of the calibrated radiance of Aqua MODIS bands 33, 35, and 36 using simultaneous nadir observations from the Atmospheric Infrared Sounder (AIRS), which is also onboard the Aqua satellite. Our analysis results show that the differences between AIRS and Aqua MODIS can be controlled well within the fluctuation range compared to the periods when the BB signals for these bands are not saturated.
\end{abstract}

Keywords: Moderate Resolution Imaging Spectroradiometer (MODIS), calibration, saturation, warm-up cool-down (WUCD)

\section{INTRODUCTION}

Aqua MODIS, the second MODIS instrument of NASA's Earth Observation System, has successfully operated for over 16 years since its launch in 2002. MODIS has sixteen thermal emissive bands (TEBs) located on two separate cold focal plane assemblies (CFPA), with bands 20-25 (3.6 - 4.6 $\mu \mathrm{m}$ ) on the short- and mid-wave infrared (SMIR) focal plane and bands 27-36 (6.4-14.4 $\mu \mathrm{m})$ on the long-wave infrared (LWIR) focal plane. Both focal planes are mounted on a common cold-stage assembly and are controlled to a nominal optimum operating temperature by a radiative cooler. Aqua MODIS uses the B-side electronics configuration and the SMIR FPA is actively controlled at $83 \mathrm{~K}$. Each TEB has a spatial resolution of $1 \mathrm{~km}$ at nadir and 10 detectors aligned in the along-track direction, with a total of $10 \mathrm{x} 1354$ samples collected per scan in the cross-track direction.

The MODIS TEBs are calibrated every scan using observations of an onboard blackbody (BB) calibrator and a space view (SV) port. The BB serves as a known radiance source with its temperature maintained at $285 \mathrm{~K}$ in normal operations. The BB temperature $\left(\mathrm{T}_{\mathrm{bb}}\right)$ is well controlled and the SV observation is used to provide a zero reference for thermal background and electronic offset. Blackbody warm-up and cool-down (WUCD) cycles, which are currently scheduled four times a year, provide detector responses over a range of temperatures from instrument ambient ( 270 K) to $315 \mathrm{~K}$. The data collected during the BB WUCD cycle is used to monitor the relationship between the radiance and the digital response and is used to track the on-orbit change of the non-linear calibration coefficients ${ }^{1,2}$.

For Aqua MODIS, there are several special scenarios that need to be considered in its TEB calibration. For bands 3132, the offset and non-linear terms are determined using on-orbit BB WUCD data rather than prelaunch data due to a 
change made to their electronic gains right before launch ${ }^{2}$. Bands 33, 35 and 36 have low saturation temperatures (Tsat), which will cause saturation when the BB temperature is above their Tsat values during the BB WUCD cycles. Regular scan-by-scan calibration of these three TEBs cannot be performed during the saturation period. Because of this, default (or fixed) linear calibration coefficients were used when the BB temperature is above their Tsat values. Due to a loss of its radiative cooler margin starting from 2006, the CFPA temperature $\left(\mathrm{T}_{\text {CFPA }}\right.$ ) showed gradually increased variations from its nominally controlled operating temperature and the magnitude of its fluctuations reached the maximum in 2013. The major impact of the $\mathrm{T}_{\text {CFPA }}$ drift is on the gains of the TEB photoconductive (PC) bands 31-36. To account for the impact of the increased variation in $\mathrm{T}_{\mathrm{CFPA}}$ on the gain during the saturation for bands 33, 35, and 36, the MODIS Characterization Support Team has developed an approach to apply a $\mathrm{T}_{\mathrm{CFPA}}$ dependent correction to the fixed calibration coefficients for the saturated scans during the WUCD ${ }^{3}$. This $\mathrm{T}_{\text {CFPA }}$ dependent gain estimation has been implemented in the Aqua MODIS Collection 6 (C6) and C6.1 L1B products.

Simultaneous nadir observations (SNOs) from the Atmospheric Infrared Sounder (AIRS), which is also onboard the Aqua satellite, have been used in the assessment of Aqua MODIS radiance observation ${ }^{4}$. In this paper, the quality of the calibrated radiance for Aqua MODIS Bands 33, 35, and 36 will be evaluated during WUCD cycle, especially for the period when their responses to the BB saturate. The MODIS TEB onboard calibrator data and calibration strategies will also be addressed in this paper. Our results show that the current calibration algorithm implemented in the C6 and C6.1 can provide the same quality of L1B data during the saturation period as the regular calibration.

Section 2 of this paper introduces the standard calibration methodology used in C6 and C6.1 L1B products for Aqua MODIS TEB. Section 3 reviews and analyzes the special calibration approach applied to the Aqua MODIS bands 33, 35, and 36. The methodology and the results of inter-comparisons with Aqua AIRS measurements are shown in Section 4.

\section{MODIS TEB CALIBRATION}

In the MODIS TEB calibration, the relationship between radiance and the digital response of a detector is described by a quadratic formulation. The top of atmosphere radiance is calculated using the following equation.

$$
L_{E V}=\frac{1}{R V S_{E V}}\left[a_{0}+b_{1} \cdot d n_{E V}+a_{2} \cdot d n_{E V}^{2}-\left(R V S_{S V}-R V S_{E V}\right) \cdot L_{S m}\right],
$$

where RVS represents response versus scan-angle; $L$ is spectral band-averaged radiance in $\mathrm{W} /\left(\mathrm{m}^{2} \mathrm{sr} \mu \mathrm{m}\right)$; $d n$ is background corrected digital count; $E V$ and $S M$ subscripts denote the earth view and scan mirror, respectively. $a_{0}, b_{1}$, and $a_{2}$ are calibration coefficients for each band, detector, and mirror side. $a_{0}$ and $a_{2}$ are provided via offline look-up tables (LUTs) and are updated if needed according to the quarterly BB WUCD measurements. $b_{1}$ is calculated from BB view data on a scan-by-scan basis by

$$
b_{1}=\left\{R V S_{B B} \cdot\left[L_{B B} \cdot \varepsilon_{B B}+\left(1-\varepsilon_{B B}\right) \cdot \varepsilon_{c a v} \cdot L_{c a v}\right]+\left(R V S_{S V}-R V S_{B B}\right) \cdot L_{s m}-a_{0}-a_{2} \cdot d n_{B B}^{2}\right\} / d n_{B B},
$$

where $\varepsilon$ represents emissivity and "cav" represent cavity. Band 21, a low gain fire detection band at 3.929-3.989 $\mu \mathrm{m}$, uses a fixed gain. Over the lifetime of Aqua MODIS, several modifications and improvements to the calibration algorithms have been applied to handle some specific issues, such as on-orbit calibration LUT update for bands 31-32, saturation of bands 33,35 , and 36 when $\mathrm{BB}$ temperature is higher than their Tsat values, and impact of $\mathrm{T}_{\mathrm{CFPA}}$ temperature drift ${ }^{2,5}$.

\section{SPECIAL CONSIDERATIONS FOR AQUA MODIS BANDS 33, 35, AND 36}

\subsection{Low saturation temperature}

It has been noticed that Aqua MODIS bands 33, 35 and 36 have relatively low saturation temperatures compared to the other TEBs, with their BB view data saturating as $\mathrm{T}_{\mathrm{bb}}$ increases ${ }^{6}$. These three Aqua TEBs show a $\mathrm{T}_{\mathrm{bb}}$ limit, Tbb_sat, during the $\mathrm{BB}$ warm-up periods above which the $\mathrm{dn}_{\mathrm{BB}}$ no longer increases. Figure 1 shows the trending of the $\mathrm{T}_{\mathrm{bb} \_}$sat for mirror side 1 with seasonal oscillations. Using on-orbit data, the Tsat are set to 293K, 296K, and 301K, which are used as thresholds (called Tsat) for bands 33, 35, and 36, respectively. They are slightly below the lowest $\mathrm{T}_{\mathrm{bb} \_}$sat over 
the mission. Since BB data from these bands during saturation periods is not usable for the scan-by-scan calibration, the $b_{1}$ coefficient (default $b_{1}$ ) needs to be determined alternatively for the calibration of earth view data.
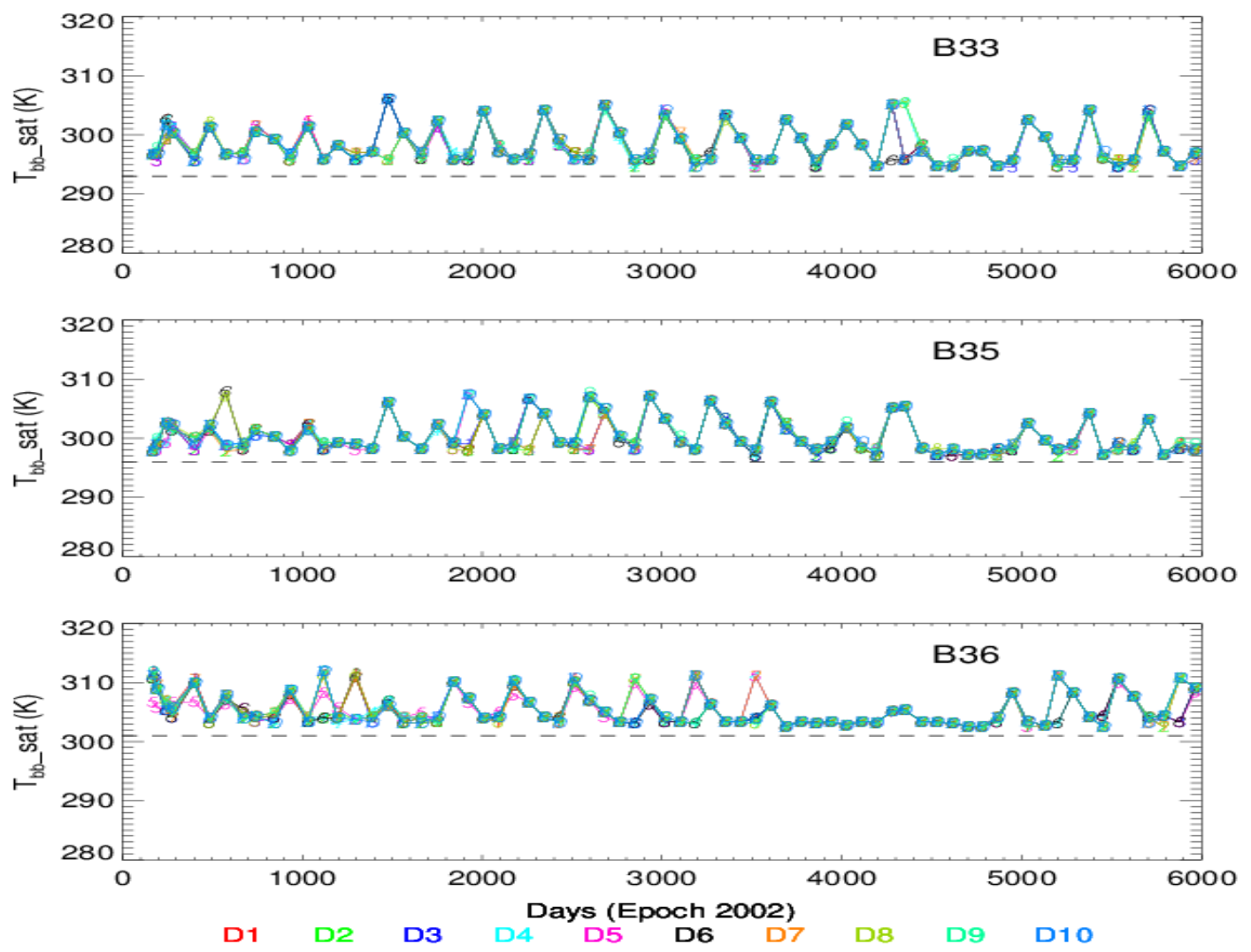

Figure 1 Entire-mission Tb__sat trending for mirror side 1.

\subsection{Review of default $b_{1}$ algorithms}

Whenever the $\mathrm{T}_{\mathrm{bb}}$ is greater than the Tsat, there is no usable $\mathrm{BB}$ data from bands 33 , 35, and 36 for the scan-by-scan calibration due to saturation. In this case, the default $b 1$ is used to calibrate the earth view data. The default $b_{1}$ algorithm has been improved from the use of a fixed $b_{1}$ value to a $T_{\text {CFPA }}$-dependent $b_{1}$. The default $b 1$ is given via the calibration LUTs on a basis of band, detector, and mirror side.
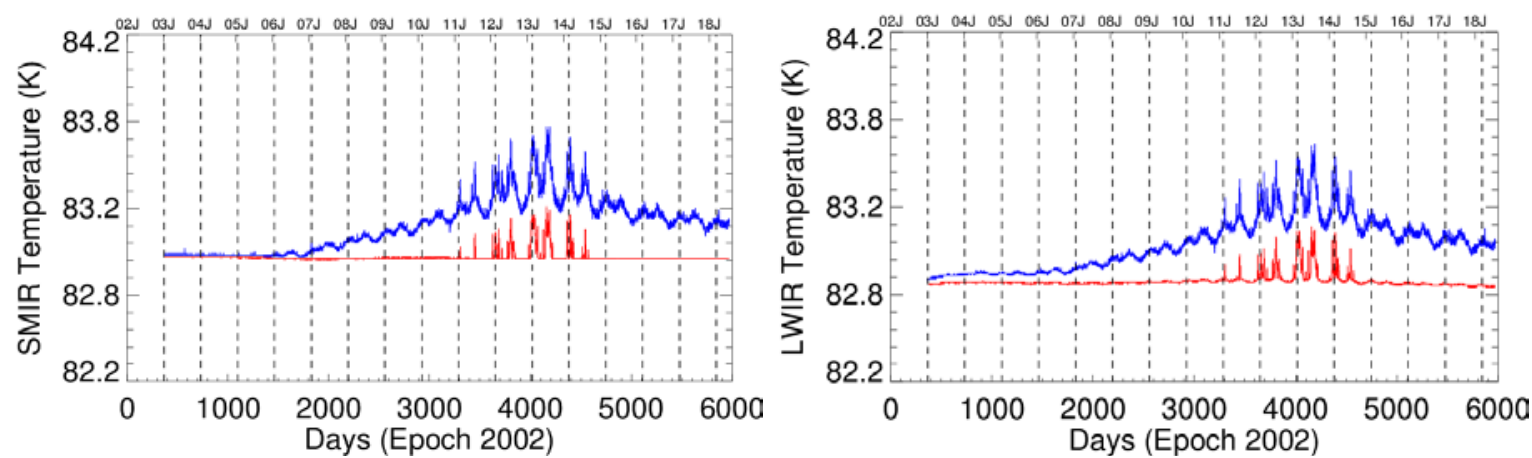

Figure 2 Aqua MODIS TCFPA trending for both the maximum (blue) and the minimum (red) temperatures. (J represents the first day of a year ) 
The default b1 with a fixed $b_{1}$ value (known as $b_{1}$, Tsat) was determined based on the $b_{1}$ value before saturation during a BB warm-up period. $b_{1}$, Tsat is the average of the $b_{1}$ values when $T_{b b}$ is in the range of Tsat $\pm 0.25 \mathrm{~K}$. The $b_{1}$, Tsat $L U T$ was regularly updated along with the $a_{0}$ and $a_{2}$ LUTs. This algorithm with a fixed value of the default $\mathrm{b}_{1}$ LUT had been used in Aqua MODIS C6 calibration algorithm until mid-2011.

For the Aqua MODIS instrument, the SMIR FPA heater average voltage has gradually decreased since launch. Since 2006, there have been instances when the heater voltage drops to zero resulting in no active temperature control for SMIR FPA ${ }^{5}$, which caused the $T_{\text {CFPA }}$ to fluctuate along with the instrument environment temperature. Figure 2 illustrates the $T_{\text {CFPA }}$ changes over time, both SMIR CFPA and LWIR CFPA. The magnitude of their fluctuations reached a maximum in 2013. It has been found that the $\mathrm{T}_{\text {CFPA }}$ fluctuations have impact mainly on the LWIR bands 31-36 ${ }^{7}$. To account for the impact of the increased $\mathrm{T}_{\mathrm{CFPA}}$ changes on the default $\mathrm{b} 1$, the MODIS Characterization Support Team

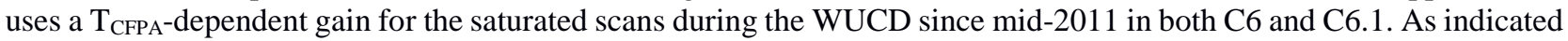
in [7], there is a strong linear relationship between $T_{\text {CFPA }}$ and band average $b_{1}$. This indicates that the major impact of the $\mathrm{T}_{\mathrm{CFPA}}$ change is captured by the corresponding change in b1. In the Aqua MODIS Collection 6 calibration algorithm, a linear correction is used to account for the CFPA influence on the digital count (dn), which is also used in the estimation of the non-linear calibration coefficient. This corrected dn can be estimated by

$$
d n_{\text {corrected }}=d n \cdot\left(1+c_{1} \cdot \Delta T_{C F P A}\right)
$$

where $c_{1}$ refers to the linear relation between the change of $b_{1}$ as a function of $\mathrm{T}_{\text {CFPA. }} \Delta \mathrm{T}_{\text {CFPA }}$ is the difference between the $\mathrm{T}_{\mathrm{CFPA}}$ and the designed operating temperature.

The observed fluctuations in $T_{\text {CFPA }}$ cause the orbital fluctuation in $b_{1}$ for bands 33 , 35, and 36 under nominal $B B$ operating conditions $(285 \mathrm{~K})$. These fluctuations can vary as high as $3 \%^{8}$ and provide a large uncertainty in the $b_{1}$, Tsat estimate. The MODIS Characterization Support Team (MCST) currently uses a correction that accounts for both the FPA temperature drift and the orbital fluctuation in $b_{1}$, Tsat to minimize the error of the default $b_{1}$ during the saturation period. For each scan, when the $\mathrm{T}_{\mathrm{bb}}$ is greater than the Tsat value, the default $\mathrm{b}_{1}$ is calculated by

$$
b_{1, \text { default }}=b_{1, \text { baseline }} \cdot\left[1+c_{1} \cdot\left(T_{\text {lwir }}-T_{\text {baseline }}\right)\right] \text {, }
$$

where

$$
b_{1, \text { baseline }}=\frac{b_{1, \text { Tsat }}}{1+c_{1} \cdot\left(T_{\text {lwir }, \text { Tsat }}-T_{\text {baseline }}\right)} .
$$

In Eq. 4-5, $T_{\text {baseline }}$ is the baseline temperature of the LWIR FPA ( $T_{\text {lwir }}$ ), which is currently set at $83 \mathrm{~K}$ for bands 33 , 35 , and 36; $T_{\text {lwir,Tsat }}$ is the average $T_{\text {lwir }}$ when $b_{1, \text { Tsat }}$ is estimated. In the C6 calibration, $T_{\text {baseline, }} b_{1}$, baseline, and $c_{1}$ are provided as default $\mathrm{b}_{1}$ LUTs in the earth view data calibration, in which $b_{1}$, baseline and $c_{1}$ LUTs are estimated using three orbits of data before each WUCD event and updated periodically.

\subsection{TCFPA-dependent default $b_{1}$ trending}

The $\mathrm{T}_{\mathrm{CFPA}}$-dependent default $\mathrm{b}_{1}$ algorithm has a significant improvement on the calibration during $\mathrm{BB}$ data saturation period compared to the fixed default $\mathrm{b}_{1}{ }^{8}$. It has been implemented in the Aqua C6 L1B since year mid-2011. Figure 3

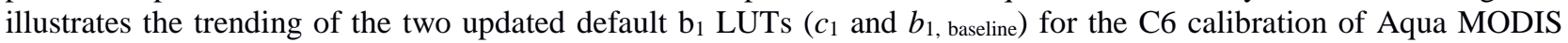
bands 33, 35, and 36. From mid-2011 until 2013, the $c_{1}$ used in Eq. 3 is not based on the OBC data right before the WUCD cycle, that is, the $c_{1}$ is obtained from the OBC data at the earlier WUCD time. Therefore, there is a noticeable

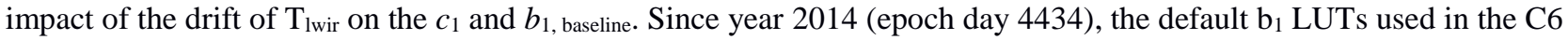
L1B product are calculated using 3-orbit OBC data before each WUCD and are updated before the WUCD event. This approach has been used in the C6.1 L1B product after July 2009. The trending of the default $b_{1}$ LUTs using this method are shown in Figure 4. In Figures 3-4, the first two $T_{\text {CFPA }}$ dependent default $b_{1}$ LUTs (01/01/2002 and 07/23/2009, epoch day 0 and 2761) are set to the same values derived from 7/23/2009 OBC data. Figures 2-4 show the $T_{\text {CFPA }}$ drift has more of impact on $c_{1}$ than $b_{1}$, baseline. By updating the default $\mathrm{b}_{1}$ LUTs ( $c_{1}$ and $b_{1}$, baseline) right before each WUCD, the T $\mathrm{T}_{\text {CFPA }}$ drift impact can be minimized therefore improving the calibration accuracy. The improvements as a result of this calibration algorithm will be demonstrated by the results of the inter-comparison with the AIRS in Section 4. 

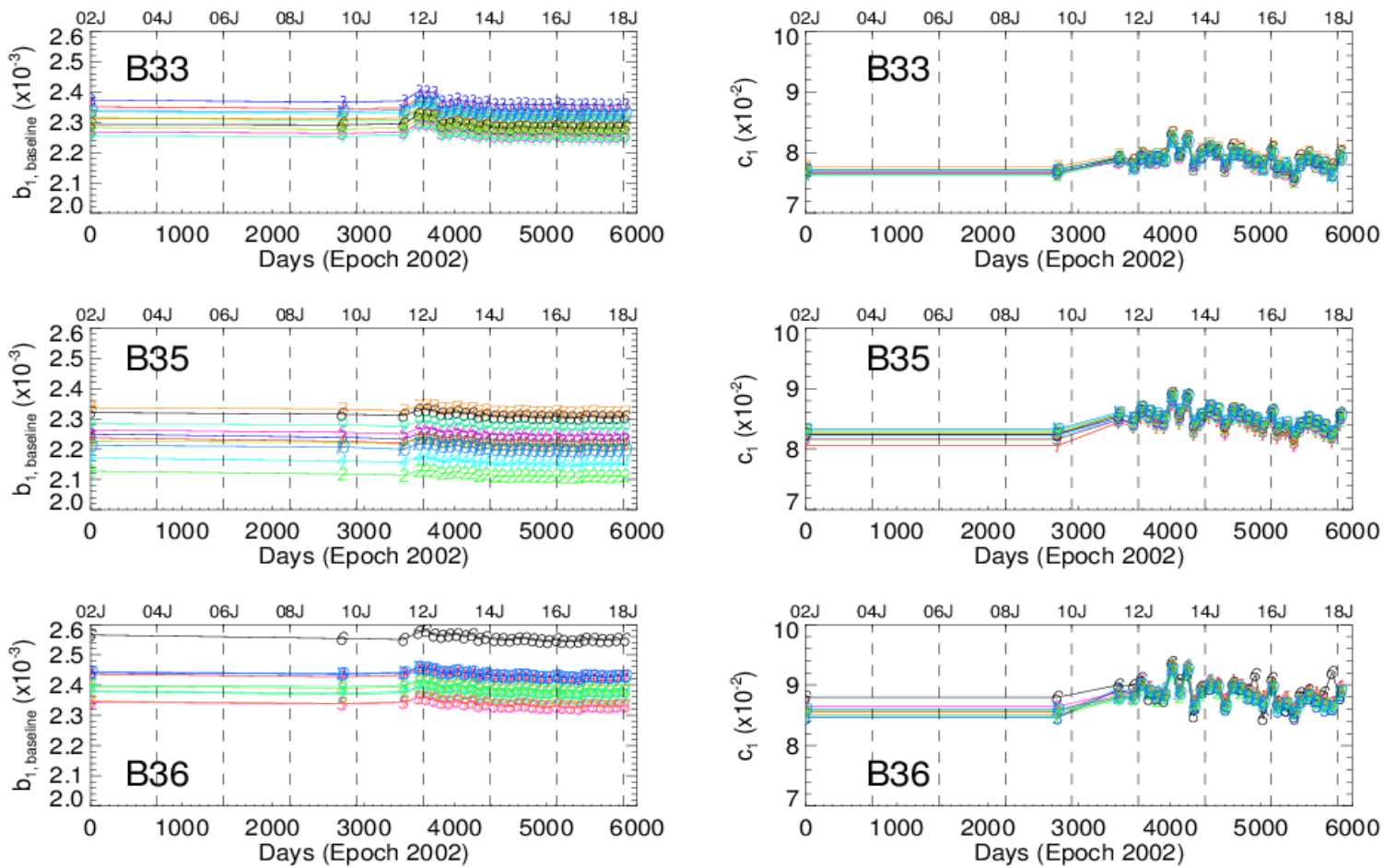

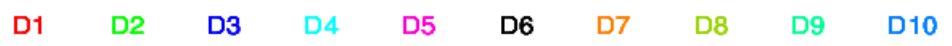

Figure 3 Trending of the default $\mathrm{b}_{1}$ LUTs in the C6 L1B. The x-axis on the top marks the first day of the year. (left: $b_{1}$, baseline; right: $c_{1}$ )
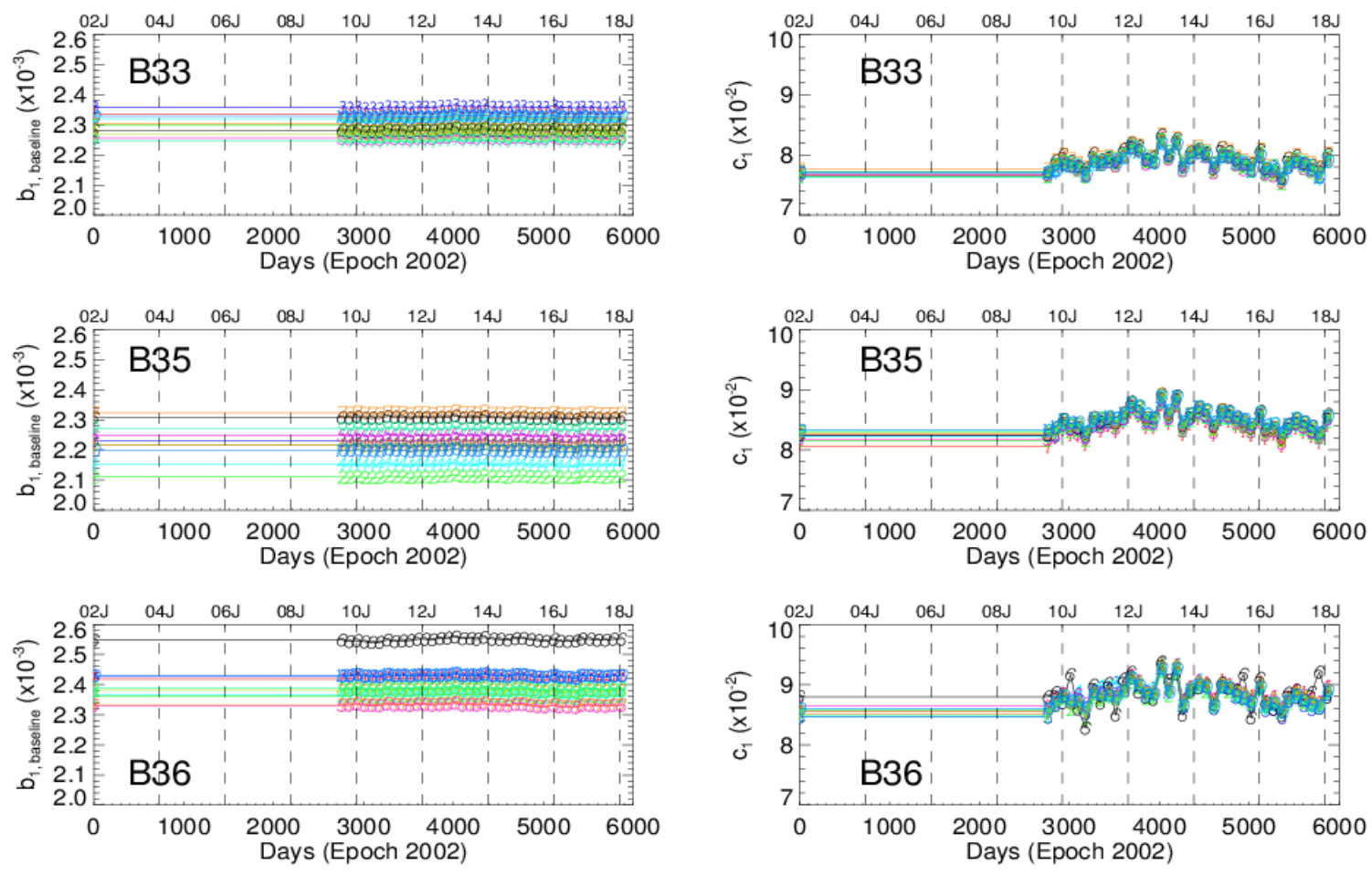

$\begin{array}{llllllllll}\mathrm{D} 1 & \mathrm{D} 2 & \mathrm{D} 3 & \mathrm{D} 4 & \mathrm{D} 5 & \mathrm{D} 6 & \mathrm{D} 7 & \mathrm{D} 8 & \mathrm{D} 9 & \mathrm{D} 10\end{array}$

Figure 4 Trending of the default $\mathrm{b}_{1}$ LUTs(left: $b_{1}$, baseline; right: $c_{1}$ ) in the C6.1 L1B. The $\mathrm{x}$-axis on the top marks the first day of the year. 


\section{EVALUATING THE CALIBRATION OF AQUA MODIS BANDS 33, 35, AND 36 DURING BB WUCD}

This evaluation of the calibration accuracy of the Aqua MODIS bands 33, 35, and 36 during WUCD was performed through the inter-comparison of MODIS and AIRS using the data from their SNO. Since MODIS and AIRS are both onboard the Aqua satellite, all of MODIS granules and the corresponding AIRS granules spanning the entire duration of the WUCD event were used for this inter-comparison. Six AIRS footprints near nadir with $13.5 \mathrm{~km}$ spatial resolution were chosen for this analysis. For each AIRS footprint, all of the MODIS pixels within a $6.75 \mathrm{~km}$ radius from the center of footprint were selected. Only the footprints with more than 100 MODIS samples in each were used in the SNO intercomparison. The hyperspectral AIRS data (2378 channels from $3.75-15.4 \mu \mathrm{m}$ ) were convolved spectrally to each of MODIS bands based on their relative spectral response (RSR). Since MODIS has spatial resolution higher than AIRS, MODIS data were aggregated to match each AIRS footprint in spatial. The details of extracting Aqua MODIS and AIRS SNO data can be found in [9].

Four WUCD events throughout the Aqua mission were chosen to evaluate the performance of the default $b_{1}$ algorithm. The first is from the early mission, 07/28/2003 (epoch day 574) WUCD, when there is no $\mathrm{T}_{\text {CFPA }}$ drift present. The second is from the mid-mission, 10/12/2012 (epoch day 3938). For this WUCD cycle, there is a large $T_{\text {CFPA }}$ drift and fluctuation (see Figure 2), and the two default $\mathrm{b}_{1}$ LUTs ( $c_{1}$ and $b_{1}$, baseline) were not updated right before the WUCD in C6 and were updated right before the WUCD in C6.1. The third and the forth WUCD are more recent, 09/17/2016 (epoch day 5374) and 11/05/2017 (epoch day 5788), with a less $\mathrm{T}_{\text {CFPA }}$ drift and the two default $\mathrm{b}_{1}$ LUTs were updated right before the WUCD events in both C6 and C6.1. In the following discussion, the radiance of a MODIS band was converted to brightness temperature (BT) using the Planck function based on the center wavelength of the spectral band.
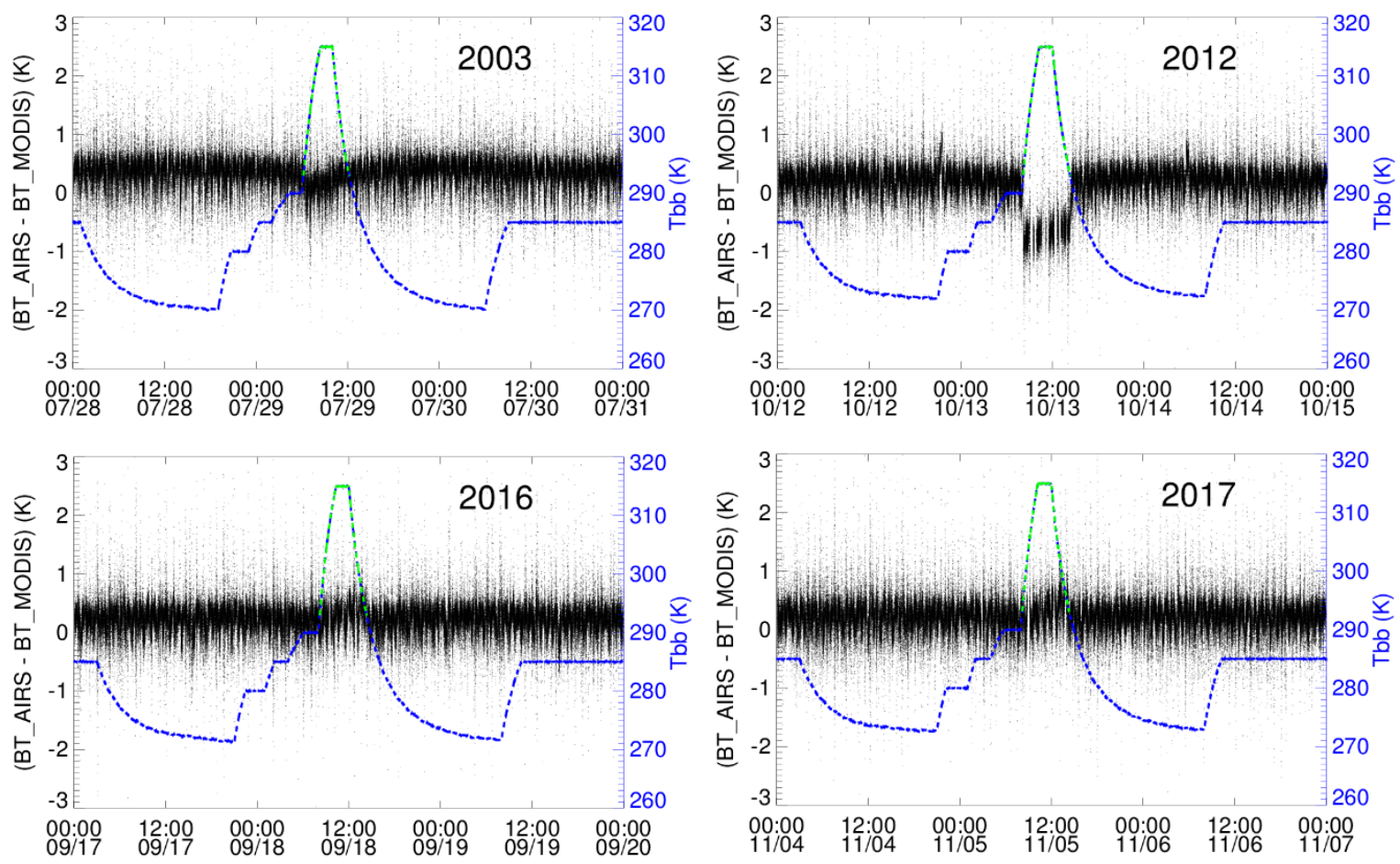

Figure 5 AIRS-MODIS SNO inter-comparison time series for Aqua MODIS C6 band 33 during WUCD events.

(Green is the period when $\mathrm{T}_{\mathrm{bb}}>$ Tsat.)

Figure 5 shows AIRS-MODIS(C6) SNO inter-comparison time series during the four WUCD periods. The $c_{1}$ and $b_{1}$, baseline LUTs without an update in time cause observed biases for the 07/28/2003 and 10/12/2012 WUCD events, The biases seen for the 2003 WUCD are due to the use of LUTs derived from July 2009 OBC data, while the biases seen during the October 2012 WUCD are caused from the use of LUTs derived before the previous WUCD event in which 
there was significant fluctuation in $\mathrm{T}_{\text {CFPA }}$. When the two default $\mathrm{b}_{1}$ LUTs were updated right before the WUCD events (see cases on 09/17/2016 and 11/05/2017), the BT differences between AIRS and MODIS during the B33 saturation period (see $\mathrm{T}_{\mathrm{bb}}$ in green) can be controlled well within the expected fluctuation compared to the periods when the BB data for these bands are not saturated. Table 1 indicates the average BT differences between AIRS and MODIS C6 measurements during the saturated and unsaturated periods for bands 33, 35, and 36 as well as the relative changes of the BT differences in the four WUCD events.

Table 1 The average BT differences (K) between AIRS and MODIS (C6) measurements during the saturated (Sat.) and unsaturated (Unsat.) periods.

\begin{tabular}{|c|c|c|c|c|c|}
\hline Band & Unsat. & \multicolumn{2}{|c|}{ Sat. } & \multicolumn{2}{|c|}{ (Sat - Unsat.) / Unsat. } \\
\hline \multicolumn{6}{|c|}{ 07/28/2003 WUCD Event } \\
\hline 33 & $0.34 \pm 0.28$ & \multicolumn{2}{|c|}{$0.16 \pm 0.28$} & \multicolumn{2}{|c|}{-0.53} \\
\hline 35 & $-0.63 \pm 0.28$ & \multicolumn{2}{|c|}{$-0.80 \pm 0.31$} & \multicolumn{2}{|c|}{0.28} \\
\hline 36 & $-0.95 \pm 0.33$ & \multicolumn{2}{|c|}{$-1.17 \pm 0.35$} & \multicolumn{2}{|c|}{0.22} \\
\hline \multicolumn{6}{|c|}{ 10/12/2012 WUCD Event } \\
\hline & & C6 & C6.1 & C6 & C6.1 \\
\hline & & & & & \\
\hline 33 & $0.21 \pm 0.27$ & $-0.66 \pm 0.33$ & $0.26 \pm 0.34$ & -4.2 & 0.21 \\
\hline 35 & $-0.65 \pm 0.28$ & $-1.13 \pm 0.31$ & $-0.73 \pm 0.39$ & 0.72 & 0.14 \\
\hline 36 & $-1.00 \pm 0.37$ & $-1.53 \pm 0.39$ & $-1.12 \pm 0.38$ & 0.52 & 0.13 \\
\hline \multicolumn{6}{|c|}{ 09/17/2016 WUCD Event } \\
\hline 33 & $0.21 \pm 0.28$ & \multicolumn{2}{|c|}{$0.27 \pm 0.29$} & \multicolumn{2}{|c|}{0.27} \\
\hline 35 & $-0.55 \pm 0.24$ & \multicolumn{2}{|c|}{$-0.47 \pm 0.31$} & \multicolumn{2}{|c|}{-0.16} \\
\hline 36 & $-0.98 \pm 0.32$ & \multicolumn{2}{|c|}{$-0.95 \pm 0.36$} & \multicolumn{2}{|c|}{-0.03} \\
\hline \multicolumn{6}{|c|}{ 11/05/2017 WUCD Event } \\
\hline 33 & $0.21 \pm 0.29$ & \multicolumn{2}{|c|}{$0.28 \pm 0.32$} & \multicolumn{2}{|c|}{0.33} \\
\hline 35 & $-0.57 \pm 0.24$ & \multicolumn{2}{|c|}{$-0.52 \pm 0.26$} & \multicolumn{2}{|c|}{-0.09} \\
\hline 36 & $-0.98 \pm 0.35$ & \multicolumn{2}{|c|}{$-1.02 \pm 0.36$} & \multicolumn{2}{|c|}{0.04} \\
\hline
\end{tabular}

The default $\mathrm{b}_{1}$ LUTs ( $c_{1}$ and $b_{1}$, baseline) highly depend on the status of the instrument (such as $\mathrm{T}_{\mathrm{CFPA}}$ ) and therefore need to be characterized in time in order to be applied to the L1B calibration. In Aqua C6.1, the default $\mathrm{b}_{1}$ LUTs are updated right before each WUCD events after 07/2009. The improvement of C6.1 comparing with C6 from 10/12/2012 WUCD data inter-comparison is illustrated in Figure 6 and also seen in Table 1.

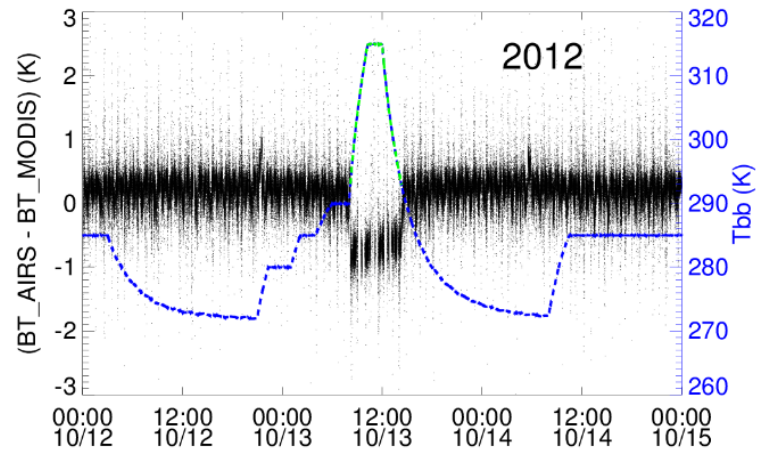

(a) B33 C6

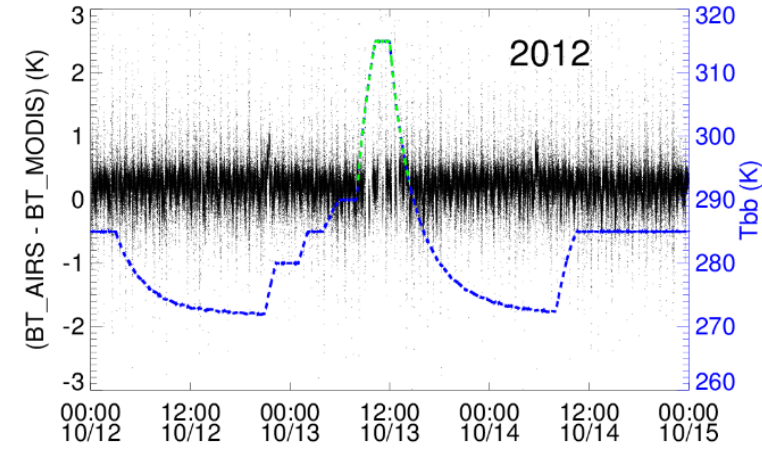

(b) B33 C6.1

Figure 6 Improvement of C6.1 for Aqua MODIS band 33, 35, and 36 for the WUCD event on 10/12/2012 (continue). (Green is the period when $\mathrm{T}_{\mathrm{bb}}>$ Tsat.) 


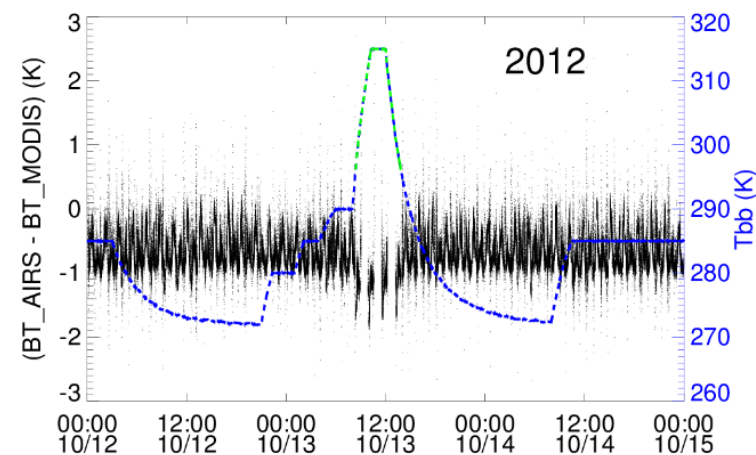

(c) B35 C6

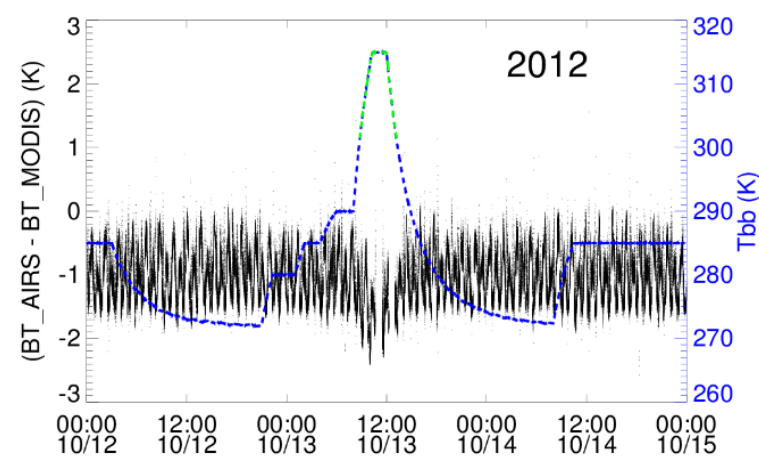

(a) B36 C6

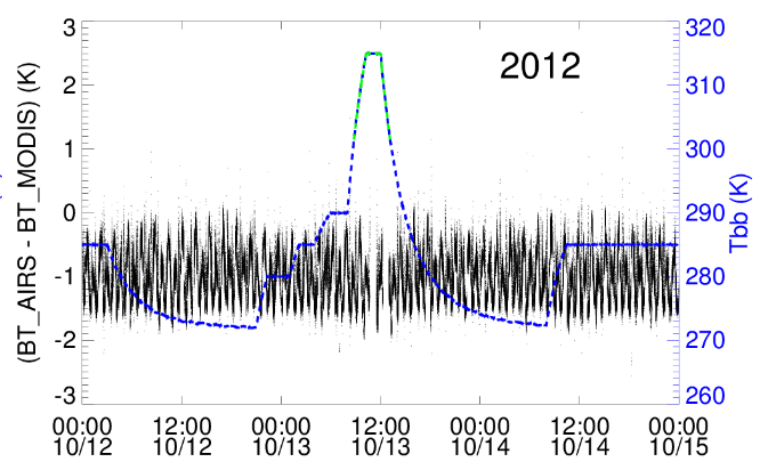

(d) B35 C6.1

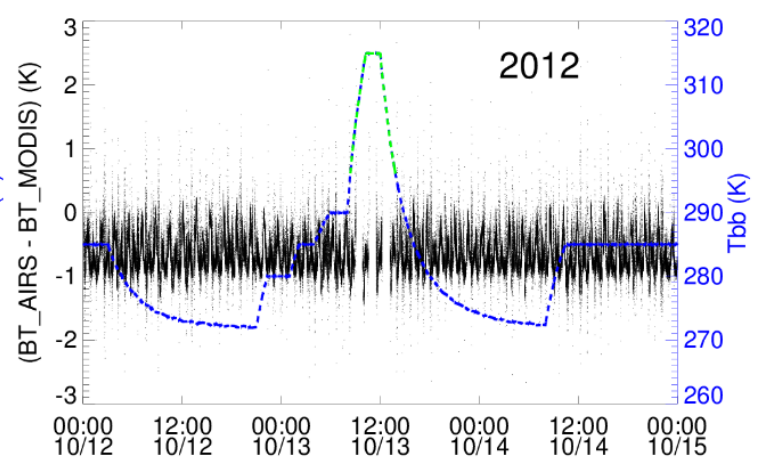

(b) B36 C6.1

Figure 6 Improvement of C6.1 for Aqua MODIS band 33, 35, and 36 for the WUCD event on 10/12/2012.

(Green is the period when $\mathrm{T}_{\mathrm{bb}}>$ Tsat.)

\section{SUMMARY}

This paper evaluates the quality of Aqua MODIS L1B calibrated data of bands 33, 35, and 36 during WUCD events.

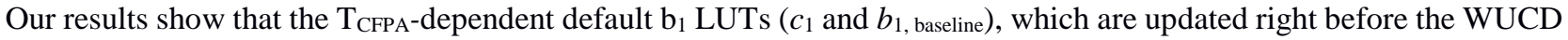
event, can eliminate the impact of the observed drift in the L1B calibration quality. Aqua C6.1 has been using this calibration approach consistently throughout the mission. Our evaluation using SNOs from the Atmospheric Infrared Sounder (AIRS), which is also onboard the Aqua satellite, shows the differences between Aqua MODIS and AIRS can stay in the same range compared to the periods when the BB signals for these bands are not saturated.

\section{ACKNOWLEDGEMENT}

The authors would like to thank Emily Aldoretta and Amit Angal for their suggestions and comments. They would also like to thank other members in the MCST TEB group for their contributions on the on-orbit calibration and characterization.

\section{REFERENCES}

[1] Xiong, X., Chiang, K., Sun, J., Che, N., and Barnes, W., “Aqua MODIS First Year On-orbit Calibration and Performance,” Proc. SPIE 5234, 391-399 (2004). 
[2] Xiong, X., Wenny, B., Wu, A., and Salomonson, V., “Aqua MODIS Thermal Emissive Bands On-orbit Calibration, Characterization, and Performance,” IEEE Trans. on Geoscience and Remote Sensing, 47(3), 803-814 (2009).

[3] Wang, Z., Xiong, X., Wu, A., Chang, T., "Update on the status of Aqua MODIS cold focal plane assembly temperature fluctuation,” Proc. SPIE 10402, 104021R (2017).

[4] Veglio, P., Tobin, D.C., Dutcher, S., Quinn, G., and Moeller, C.C., "Long-term assessment of Aqua MODIS radiance observation using comparisons with AIRS and IASI,” J. Geophys. Res. Atmos., 121, 8460-8471 (2016).

[5] Wang, Z., Madhavan S., Xiong X., Wu A., and Wenny B.N., "Monitoring and assessment of the temperature fluctuation of Aqua MODIS cold focal plane assembly,” Proc. SPIE 8510, 85100K (2012).

[6] Xiong, X., Chiang, V., Chen, N., and Barnes, W., "Aqua MODIS thermal emissive bands calibration algorithm and preliminary results,” Proc. SPIE 4814, 255-263 (2002).

[7] Xiong X., Wu, A., Wenny, B.N., Madhavan, S., Wang, Z., Li, Y., Chen, N., Barnes, W.L., and Salomonson, V.V., "Terra and Aqua MODIS Thermal Emissive Bands On-Orbit Calibration and Performance," IEEE Trans. on Geoscience and Remote Sensing, 53(10), 5709-5721 (2015).

[8] Wenny, B.N., Wu, A., Madhavan, S., Wang, Z., Li, Y., Chen, N., Chiang, K., and Xiong, X., "MODIS TEB Calibration Approach in Collection 6," Proc. SPIE 8533, 85331M-7 (2012).

[9] Li Y., Wu A., Wenny B.N., and Xiong X., "Evaluating the Impact of Cold Focal Plane Temperature on Aqua MODIS Thermal Emissive Band Calibration,” Proc. SPIE 9607, 96070U (2015). 